\title{
NONLINEAR FRACTURE ANALYSIS OF PIEZOELECTRIC CERAMICS BY FINITE ELEMENT METHOD
}

\author{
K.Y.Sze , Y.-S.Pan* \\ Department of Mechanical Engineering, The University of Hong Kong \\ Pokfulam Road, Hong Kong SAR, P.R.CHINA
}

\begin{abstract}
In this paper, a simple nonlinear constitutive model for piezoelectric ceramics is devised. The model is implemented in a research finite element code and used to study the effect of domain switching and electric nonlinearity on the cracking behaviour. J-integrals are computed along contours close to and well away from the crack tip. For linear materials, the computed J-integrals are essentially path independent. With the material non-linearity considered, the integrals computed at the crack tip vicinity is higher than the ones computed well away from the crack tip. Using the former as the fracture criterion, it is found that crack propagation can be promoted by the electric field.
\end{abstract}

keywords : electric nonlinearity, polarization switching, finite element, piezoelectricity, fracture

Published in Engineering Fracture Mechanics, 68, 1335-1351 (2001)

\footnotetext{
* on leave from Institute of Computational Engineering Sciences, Southwest Jiaotong University
} 


\section{INTRODUCTION}

Piezoelectric ceramics are widely used in smart structure, actuator and transducer technologies. For control effectiveness and high strain output, large electric fields are often applied to the materials. It is noteworthy that piezoelectric ceramics exhibit a strong non-linearity associated with domain wall motion which is also known as domain or polarization switching [1]. When operated under strong electric fields, piezoelectric ceramics may be depolarized and fractured. Thus, their actuating function will be seriously degraded. Consequently, fatigue and fracture behaviors of the materials under strong electric fields have attracted considerable interest [2-21].

In theoretical analyses of cracks in piezoelectric ceramics, solutions based on linear fracture mechanics have been obtained [6-10,18-19]. However, the influence of strong electric fields on the fracture behavior remains to be an outstanding issue. For the poling direction parallel to the applied remote electric field and tensile stress but perpendicular to crack face, experimental results reveal that positive (along the poling direction) and negative electric fields foster and inhibit crack propagation, respectively [11]. On the other hand, the energy release rate derived from linear piezoelectric fracture mechanics indicates that crack propagation is always impeded by the electric field no matter the latter is positive or negative. Researchers have attempted to explain the experimental observation by changing the boundary conditions at the crack surface. McMeeking [4], Dunn [10] and Zhang, Qian \& Tong [19] employed a permeable condition on the surface instead of the impermeable condition. With the permeable condition, the electric displacement singularity arising from the impermeable condition disappears and the energy release rate becomes independent of the applied electric field. Meanwhile, Kumar \& Singh [17] found by finite element analysis that the traction-free boundary condition on the crack surfaces can be invalid for two combinations of electric and mechanical loads : (i) negative electric field and negative applied stress; (ii) positive electric field and positive applied strain. To account for the non-traction-free boundary conditions, a contact model was proposed. However, the computed energy release rate still cannot explain the experimental observation. Park \& Sun [11] argued that crack propagation is a mechanical process and the strain (mechanical) energy release rate instead of the total (electromechanical) energy release rate should be used as the fracture criterion. Their finite element results are in reasonably agreement with the experimental results. However, the correctness of their finite element results was questioned by Kumar \& Singh recently $[15,16]$.

Gao and his coworkers $[13,14]$ pointed out that electric ductility should not be ignored in formulating a fracture criterion. An electric saturation strip model analogous to Dugdale's model in conventional elastoplastic fracture [12-14] was proposed. The local and global energy release rates were derived by considering paths that pass and do not pass through the saturation strip, 
respectively. Under the small-scale yielding condition, the global energy release rate is equal to that evaluated by linear fracture mechanics. It was also shown that the local energy release rate is independent of the electric saturation strength and the length of the saturation strip. The local energy release rate indicates that the fracture stress is essentially a linear function of the applied electric field. Yang and his coworkers [20,21] also proposed a model that considers the effect of $90^{\circ}$ and $180^{\circ}$ polarization switchings in ferroelectric materials. The model can also yield predictions that match the aforementioned experimental cracking behavior.

On the other hand, many researchers have been working on nonlinear constitutive modeling of piezoelectric materials [22-33]. At least three categories of models have been proposed. The first category is the micro-mechanics approach in which the domain wall motions of the crystals, $90^{\circ}$ or/and $180^{\circ}$ switching, are considered but only the macroscopic effect of materials is modelled [2226]. The second category is the phenomenological hysteresis approach in which the electric displacement-electric field hysteresis loop and the strain-electric field butterfly loop are modelled by various phenomenological parameters [27-31]. The third category is based on thermodynamics approach [32-37]. It should be remarked that not many of the above models can be applied to or implemented in finite element analysis. Owing to the practical importance of finite element analysis, ad hoc nonlinear constitutive models have also been proposed recently [25,26,31,35,38].

In this paper, a simple constitutive model for piezoelectric ceramics is devised that takes electric nonlinearity and polarization switching into account. Based on the model, nonlinear finite element procedure is devised and implemented in a research code. The latter is then employed for nonlinear fracture analysis of mode I crack in piezoelectric panels. Both the local energy release rate based upon the saturation strip model $[13,14]$ and the local $J$-integral computed in the crack tip vicinity predict that the fracture stress decreases as the positive electric field increases. Compared to the former, the latter is qualitatively more consistent with the experimental results obtained by Park \& Sun [11] in the sense that the fracture load drops more gently and does not drop to zero even for very large electric field.

\section{ELECTRIC NONLINEARITY}

With the positive $x_{3}$-direction being the poling direction, the plane strain constitutive relation of linear piezoelectric material can be expressed as :

$$
\left\{\begin{array}{c}
\boldsymbol{\tau} \\
\mathbf{D}-\mathbf{D}_{o}
\end{array}\right\}=\left[\begin{array}{cc}
\mathbf{c} & \mathbf{e} \\
-\mathbf{e} & -\boldsymbol{\epsilon}
\end{array}\right]\left\{\begin{array}{c}
\boldsymbol{\gamma} \\
-\mathbf{E}
\end{array}\right\}
$$


where $\boldsymbol{\tau}=\left\{\tau_{11}, \tau_{33}, \tau_{31}\right\}^{T}$ is the vector of stress components, $\boldsymbol{\gamma}=\left\{\gamma_{11}, \gamma_{33}, 2 \gamma_{31}\right\}^{T}$ is the vector of strain components, $\mathbf{E}=\left\{E_{1}, E_{3}\right\}^{T}$ is the electric field, $\mathbf{D}=\left\{D_{1}, D_{3}\right\}^{T}$ is the electric displacement, $\mathbf{D}_{o}$ is the remnant polarization, $\mathbf{c}=\mathbf{c}^{T}$ is the elasticity matrix measured at constant electric field, $\mathbf{e}$ is the piezoelectric matrix measured at constant strain, $\boldsymbol{\epsilon}=\boldsymbol{\epsilon}^{T}$ is the dielectric matrix measured at constant strain. Moreover,

$$
\boldsymbol{\gamma}=\mathbf{L u}, \mathbf{E}=-\nabla \phi
$$

in which

$$
\begin{aligned}
& \mathbf{u}=\left\{u_{1}, u_{3}\right\}^{T} \text { is the displacement vector, } \phi \text { is the electric potential, } \\
& \mathbf{L}=\left[\begin{array}{ccc}
\partial / \partial x_{1} & 0 & \partial / \partial x_{3} \\
0 & \partial / \partial x_{3} & \partial / \partial x_{1}
\end{array}\right]^{T} \text { and } \nabla=\left[\begin{array}{l}
\partial / \partial x_{1} \\
\partial / \partial x_{3}
\end{array}\right]
\end{aligned}
$$

For the nonlinear material behaviour, Fig.1 shows the responses of a typical piezoelectric ceramics poled along the $x_{3}$-direction. Under zero stress and electric field, the electric displacement is the same as the remnant polarization, i.e. $\mathbf{D}_{o}=\left\{0, D_{o}\right\}^{T}$, as given at $b$ in the figures. When the electric field $\mathbf{E}=\left\{0, E_{3}\right\}^{T}$ is varied, the crystal stays along $a-b-c-d$ unless the electric field is strong enough to drive a $180^{\circ}$ switching which starts at $a$ and completes at $f$. After the switching, the remnant polarization changes to $\left\{0,-D_{o}\right\}^{T}$. Similarly, the crystal stays along $e-f-g-h$ unless the electric field is strong enough to drive another $180^{\circ}$ switching which starts at $h$ and completes at $c$. After the second switching, the remnant polarization changes back to $\left\{0, D_{o}\right\}^{T}$. The arrows in the figure indicate that the switching paths from $a$ to $f$ and $h$ to $c$ are irreversible. Furthermore, electric saturation [12-14,34,35,38] occurs between $c$ and $d$ as well as $f$ and $e$ in which the tangential dielectric constants are smaller than that given by the linear rule in (1). It should be remarked that the more complicated $90^{\circ}$ switching which also involves remnant strain is not considered.

Assuming that the criteria for domain switching and electric saturation are functions of the electric displacement only $[22,26]$ and can be expressed in a single function as :

$$
F(\mathbf{D}, \kappa)=0
$$

we have

$$
\left(\frac{\partial F}{\partial \mathbf{D}}\right)^{T} d \mathbf{D}=-\frac{\partial F}{\partial \kappa} d \kappa
$$

in which $\kappa$ is a parameter governing the electric saturation behaviour between $c$ and $d$ and between $f$ and $e$. Similar to the strain in associated flow rule in elastoplastic materials, the electric field can be 
partitioned into "elastic" electric field $\mathbf{E}^{e}$ and "plastic" electric field $\mathbf{E}^{p}$, i.e.

$$
\mathbf{E}=\mathbf{E}^{e}+\mathbf{E}^{p}, d \mathbf{E}=d \mathbf{E}^{e}+d \mathbf{E}^{p} .
$$

The linear piezoelectric relations still hold for $\mathbf{E}^{e}$, i.e.

$$
\left\{\begin{array}{c}
\boldsymbol{T} \\
\mathbf{D}-\mathbf{D}_{o}
\end{array}\right\}=\left[\begin{array}{cc}
\mathbf{c} & \mathbf{e} \\
-\mathbf{e} & -\boldsymbol{\epsilon}
\end{array}\right]\left\{\begin{array}{c}
\boldsymbol{\gamma} \\
-\mathbf{E}^{e}
\end{array}\right\}=\left[\begin{array}{cc}
\mathbf{c} & \mathbf{e} \\
-\mathbf{e} & -\boldsymbol{\epsilon}
\end{array}\right]\left\{\begin{array}{c}
\boldsymbol{\gamma} \\
-\left(\mathbf{E}-\mathbf{E}^{p}\right)
\end{array}\right\}
$$

Adopting $F$ as the flow potential,

$$
d \mathbf{E}^{p}=d \lambda \frac{\partial F}{\partial \mathbf{D}}
$$

By merging the last two equations, we have the following incremental relations :

$$
d \boldsymbol{\tau}=\mathbf{c} d \boldsymbol{\gamma}-\mathbf{e}^{T}\left(d \mathbf{E}-\frac{\partial F}{\partial \mathbf{D}} d \lambda\right), d \mathbf{D}=\mathbf{e} d \boldsymbol{\gamma}+\mathbf{\epsilon}\left(d \mathbf{E}-\frac{\partial F}{\partial \mathbf{D}} d \lambda\right) .
$$

After pre-multiplying with $(\partial F / \partial \mathbf{D})^{T}$ and invoking Eqn.(4), the last expression in Eqn.(8) becomes :

$$
-\frac{\partial F}{\partial \kappa} d \kappa=\left(\frac{\partial F}{\partial \mathbf{D}}\right)^{T}\left(\mathbf{e} d \boldsymbol{\gamma}+\boldsymbol{\epsilon} d \mathbf{E}-\boldsymbol{\epsilon} \frac{\partial F}{\partial \mathbf{D}} d \lambda\right)
$$

or

$$
d \lambda=\frac{1}{\chi}\left(\frac{\partial F}{\partial \mathbf{D}}\right)^{T}(\mathbf{e} d \boldsymbol{\gamma}+\mathbf{\epsilon} d \mathbf{E})
$$

in which

$$
\chi=\left(\frac{\partial F}{\partial \mathbf{D}}\right)^{T} \boldsymbol{\epsilon} \frac{\partial F}{\partial \mathbf{D}}-\frac{\partial F}{\partial \kappa} \frac{d \kappa}{d \lambda}
$$

Back substituting Eqn.(10) into Eqn.(8) results in

$$
d \boldsymbol{\tau}=\mathbf{c}^{e p} d \boldsymbol{\gamma}-\left(\mathbf{e}^{e p}\right)^{T} d \mathbf{E}, d \mathbf{D}=\mathbf{e}^{e p} d \boldsymbol{\gamma}+\boldsymbol{\epsilon}^{e p} d \mathbf{E}
$$

where

$$
\mathbf{c}^{e p}=\mathbf{c}+\frac{1}{\chi} \mathbf{e}^{T} \frac{\partial F}{\partial \mathbf{D}}\left(\frac{\partial F}{\partial \mathbf{D}}\right)^{T} \mathbf{e}, \quad \mathbf{e}^{e p}=\mathbf{e}-\frac{1}{\chi} \boldsymbol{\epsilon} \frac{\partial F}{\partial \mathbf{D}}\left(\frac{\partial F}{\partial \mathbf{D}}\right)^{T} \mathbf{e}, \boldsymbol{\epsilon}^{e p}=\boldsymbol{\epsilon}-\frac{1}{\chi} \boldsymbol{\epsilon} \frac{\partial F}{\partial \mathbf{D}}\left(\frac{\partial F}{\partial \mathbf{D}}\right)^{T} \boldsymbol{\epsilon} .
$$

are the "elastoplastic" material matrices for incremental computation.

\section{A MODEL FOR POLARIZATION SWITCHING \& ELECTRIC SATURATION}

Constitutive models for domain switching and electric saturation are still under extensive research $[22,26,34,38]$. For a preliminary study on the effect of domain switching and electric yielding on piezoceramic fracture, a simply constitutive model accounting for domain switching and electric 
saturation is here postulated after consulting the following works. Chan \& Hagood [22] and Fotinich \& Carman [26] consider the material as a collection of single crystals with a certain orientation. Domain switching is determined in a single cell of material. The lattice structure of piezoelectric crystals is treated to be tetragonal. In general, a cell can undergo $180^{\circ}$ or $90^{\circ}$ polarization switchings. On the other hand, the experimental results of Park, Carman \& Park [39-41] showed that domain switching appears to be predominately $180^{\circ}$ without any $90^{\circ}$ switching at the mesoscopic level in the stress concentration regions of a notched piezoelectric ceramics panel subjected to large electric fields. For the present preliminary study, only the $180^{\circ}$ switching is considered.

Without losing generosity, the material is assumed to be poled along the positive $x_{3}$-direction. Analogous to the elastic-perfect plastic model, electric saturation is taken to be "perfect". Hence, $c-d$ and $e-f$ in Fig.2a are horizontal. Meanwhile, polarization switching is taken to be an instantaneous process with respect to the driving electric field. Thus, $a-f$ and $h-c$ in Fig.2a are vertical. Along $a-b-c-d$ and $e-f-g-h$, the remnant polarizations are respectively $\mathbf{D}_{o}=\left\{0, D_{o}\right\}^{T}$ and $\mathbf{D}_{o}=\left\{0,-D_{o}\right\}^{T}$ which are the only alternatives in the present model. The criteria for electric saturation and domain switching are respectively :

$$
\left(\mathbf{D}-\mathbf{D}_{o}\right)^{T} \mathbf{n}_{o}>D_{c} \quad \text { and } \quad\left(\mathbf{D}-\mathbf{D}_{o}\right)^{T} \mathbf{n}_{o}<-D_{c}
$$

where $D_{c}$ is positive and $\mathbf{n}_{o}$ is the unit vector along $\mathbf{D}_{o}$. The above criteria for polarization switching are the same as the one suggested by Fotinich and Carman [26]. In the present model, domain switching is immediately followed by electric saturation for monotonic loadings. Hence,

$$
F(\mathbf{D}, \kappa)=\left(\mathbf{D}-\mathbf{D}_{o}\right)^{T} \mathbf{n}_{o}-D_{c}=\mathbf{D}^{T} \mathbf{n}_{o}-\kappa
$$

where $\kappa=\mathbf{D}_{o}^{T} \mathbf{n}_{o}-D_{c}=D_{o}-D_{c}$. Noting that $\mathbf{D}_{o}, \mathbf{n}_{o}$ and $\kappa$ are piecewise constant,

$$
\frac{\partial F}{\partial \mathbf{D}}=\mathbf{n}_{o} \quad \text { and } \quad \frac{d \kappa}{d \lambda}=0
$$

Thus, $\chi$ in Eqn.(10) is equal to $\mathbf{n}_{o}^{T} \boldsymbol{\epsilon} \mathbf{n}_{o}=\epsilon_{33}$ where $\left[\epsilon_{i j}\right]=\boldsymbol{\epsilon}$. Moreover, the "elastoplastic" material matrices in Eqn.(12) become

$$
\mathbf{c}^{e p}=\mathbf{c}+\frac{1}{\epsilon_{33}} \mathbf{e}^{T}\left[\begin{array}{ll}
0 & 0 \\
0 & 1
\end{array}\right] \mathbf{e}, \mathbf{e}^{e p}=\mathbf{e}-\frac{1}{\epsilon_{33}} \boldsymbol{\epsilon}\left[\begin{array}{ll}
0 & 0 \\
0 & 1
\end{array}\right] \mathbf{e}, \boldsymbol{\epsilon}^{e p}=\boldsymbol{\epsilon}-\frac{1}{\epsilon_{33}} \boldsymbol{\epsilon}\left[\begin{array}{ll}
0 & 0 \\
0 & 1
\end{array}\right] \boldsymbol{\epsilon} .
$$




\section{NONLINEAR FINITE ELEMENT FOMULATION}

For finite element formulation, the "virtual work" principle for piezoelectricity can be employed [42], namely

$$
\delta \Pi=\int_{\Omega}\left(\boldsymbol{\tau}^{T} \delta \boldsymbol{\gamma}-\mathbf{D}^{T} \delta \mathbf{E}-\overline{\mathbf{b}}^{T} \delta \mathbf{u}\right) d \Omega-\int_{S_{t}} \overline{\mathbf{t}}^{T} \delta \mathbf{u} d s-\int_{S_{\omega}} \bar{\omega} \delta \phi d s=0
$$

where $\Omega$ denotes the piezoelectric domain, $\delta$ is variational symbol, $\overline{\mathbf{b}}$ is the prescribed body force, $\overline{\mathbf{t}}$ is the surface traction prescribed over the boundary portion $S_{t}$ and $\bar{\omega}$ is the surface charge density prescribed over $S_{\omega}$. With displacement and electric potential taken to be the nodal d.o.f.s,

$$
\mathbf{u}=\mathbf{N}_{m} \mathbf{q}_{m}, \phi=\mathbf{N}_{e} \mathbf{q}_{e}, \boldsymbol{\gamma}=\mathbf{B}_{m} \mathbf{q}_{m}=\left(\mathbf{L} \mathbf{N}_{m}\right) \mathbf{q}_{m}, \mathbf{E}=-\left(\nabla \mathbf{N}_{e}\right) \mathbf{q}_{e}=-\mathbf{B}_{e} \mathbf{q}_{e}
$$

in which N's are the interpolation matrices, B's are self-defined, $\mathbf{q}_{m}$ and $\mathbf{q}_{e}$ are respectively the vectors containing the nodal displacements and nodal electric potential. Substituting Eqn.(17) into Eqn.(16), we have

$$
\left.\delta \Pi=\left\{\begin{array}{l}
\delta \mathbf{q}_{m} \\
\delta \mathbf{q}_{e}
\end{array}\right\}^{T}\left(\begin{array}{l}
\mathbf{P}_{m} \\
\mathbf{P}_{e}
\end{array}\right\}-\left\{\begin{array}{l}
\mathbf{f}_{m} \\
\mathbf{f}_{e}
\end{array}\right\}\right)=\mathbf{0}
$$

where

$$
\mathbf{P}_{m}=\int_{\Omega} \mathbf{B}_{m}^{T} \boldsymbol{\tau} d \Omega, \mathbf{P}_{e}=\int_{\Omega} \mathbf{B}_{e}^{T} \mathbf{D} d \Omega, \mathbf{f}_{m}=\int_{\Omega} \mathbf{N}_{m}^{T} \overline{\mathbf{b}} d \Omega+\int_{S_{t}} \mathbf{N}_{m}^{T} \overline{\mathbf{t}} d s, \mathbf{f}_{e}=\int_{S_{\omega}} \mathbf{N}_{e}^{T} \bar{\omega} d s
$$

To solve the nonlinear equation in Eqn.(22) by iterative methods, the following generalized residual force vector is defined :

$$
\left\{\begin{array}{c}
\boldsymbol{\Psi}_{m}(\mathbf{q}) \\
\boldsymbol{\Psi}_{e}(\mathbf{q})
\end{array}\right\}=\left\{\begin{array}{c}
\mathbf{P}_{m}(\mathbf{q}) \\
\mathbf{P}_{e}(\mathbf{q})
\end{array}\right\}-\left\{\begin{array}{c}
\mathbf{f}_{m} \\
\mathbf{f}_{e}
\end{array}\right\} \text { and thus }\left\{\begin{array}{c}
\boldsymbol{\Psi}_{m}(\mathbf{q}+\Delta \mathbf{q}) \\
\boldsymbol{\Psi}_{e}(\mathbf{q}+\Delta \mathbf{q})
\end{array}\right\} \approx\left\{\begin{array}{c}
\boldsymbol{\Psi}_{m}(\mathbf{q}) \\
\boldsymbol{\Psi}_{e}(\mathbf{q})
\end{array}\right\}+\mathbf{K}_{T}(\mathbf{q}) \Delta \mathbf{q}
$$

where

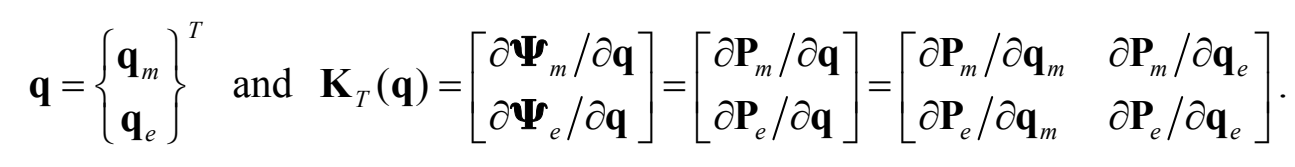

By virtue of Eqn.(11) and Eqn.(17), the generalized tangential stiffness matrix becomes

$$
\mathbf{K}_{T}(\mathbf{q})=\left[\begin{array}{cc}
\int_{\Omega} \mathbf{B}_{m}^{T} \mathbf{c}^{e p} \mathbf{B}_{m} d \Omega & \int_{\Omega} \mathbf{B}_{m}^{T}\left(\mathbf{e}^{e p}\right)^{T} \mathbf{B}_{e} d \Omega \\
\int_{\Omega} \mathbf{B}_{e}^{T} \mathbf{e}^{e p} \mathbf{B}_{m} d \Omega & -\int_{\Omega} \mathbf{B}_{e}^{T} \boldsymbol{\epsilon}^{e p} \mathbf{B}_{e} d \Omega
\end{array}\right]
$$

Based on the linear solution obtained for the total generalized load $\mathbf{f}=\left\{\mathbf{f}_{m}^{T}, \mathbf{f}_{e}^{T}\right\}^{T}$, polarization switching is checked by Eqn.(12) at each element centroid. The signs of the remnant polarization 
and the piezoelectric matrix of the element are changed accordingly.

The total generalized load $\mathbf{f}$ is then applied incrementally. Let the loads at the $n$-th and $(n+1)$-th steps be $\mathbf{f}_{n}$ and $\mathbf{f}_{n+1}$, respectively. With $\mathbf{q}_{n}$ be the converged solution at $\mathbf{f}_{n}$ and the first iterative solution for the solution $\mathbf{q}_{n+1}$ at load $\mathbf{f}_{n+1}$ be $\mathbf{q}_{n}$, i.e. $\mathbf{q}_{n+1}^{1}=\mathbf{q}_{n}$, successive iterative solution of $\mathbf{q}_{n+1}$ is :

$$
\mathbf{q}_{n+1}^{i+1}=\mathbf{q}_{n+1}^{i}+\Delta \mathbf{q}_{n}^{i}
$$

in which $\Delta \mathbf{q}_{n}^{i}$ is solved by setting Eqn.(19) to zero, i.e

$$
\Delta \mathbf{q}_{n}^{i}=-\left[\mathbf{K}_{T}\left(\mathbf{q}_{n+1}^{i}\right)\right]^{-1}\left\{\begin{array}{c}
\boldsymbol{\Psi}_{m}\left(\mathbf{q}_{n+1}^{i}\right) \\
\boldsymbol{\Psi}_{e}\left(\mathbf{q}_{n+1}^{i}\right)
\end{array}\right\} .
$$

For the purpose of computing the internal generalized forces, the following generalized strain increment is computed :

$$
\left\{\begin{array}{c}
\Delta \boldsymbol{\gamma}_{n}^{i} \\
\Delta \mathbf{E}_{n}^{i}
\end{array}\right\}=\left[\begin{array}{l}
\mathbf{B}_{m} \\
\mathbf{B}_{e}
\end{array}\right]\left(\mathbf{q}_{n+1}^{i+1}-\mathbf{q}_{n}\right) .
$$

Let $\boldsymbol{\tau}_{n}$ and $\mathbf{D}_{n}$ be the converged stress and electric displacement at the $n$-th load step. To compute the stress and electric displacement increments, the following cases have to be considered :

$\underline{\text { Case } 1}$ If $\left(\mathbf{D}_{n}-\mathbf{D}_{o}\right)^{T} \mathbf{n}_{o}<D_{c}$ and $\left(\mathbf{D}_{n}+\mathbf{e} \Delta \boldsymbol{\gamma}_{n}^{i}+\mathbf{\epsilon} \Delta \mathbf{E}_{n}^{i}-\mathbf{D}_{o}\right)^{T} \mathbf{n}_{o}<D_{c}$, the material remains "elastic", thus

$$
\boldsymbol{\tau}_{n+1}^{i+1}=\boldsymbol{\tau}_{n}+\mathbf{c} \Delta \boldsymbol{\gamma}_{n}^{i}-\mathbf{e}^{T} \Delta \mathbf{E}_{n}^{i}, \mathbf{D}_{n+1}^{i+1}=\mathbf{D}_{n}+\mathbf{e} \Delta \boldsymbol{\gamma}_{n}^{i}+\mathbf{\epsilon} \Delta \mathbf{E}_{n}^{i}
$$

Case 2 If $\left(\mathbf{D}_{n}-\mathbf{D}_{o}\right)^{T} \mathbf{n}_{o}<D_{c}$ and $\left(\mathbf{D}_{n}+\mathbf{e} \Delta \boldsymbol{\gamma}_{n}^{i}+\mathbf{\epsilon} \Delta \mathbf{E}_{n}^{i}-\mathbf{D}_{o}\right)^{T} \mathbf{n}_{o} \geq D_{c}$, the material transits from "elastic" to "plastic", thus [44]

$$
\begin{aligned}
& \boldsymbol{\tau}_{n+1}^{i+1}=\boldsymbol{\tau}_{n}+\left[m \mathbf{c}+(1-m) \mathbf{c}^{e p}\right] \Delta \boldsymbol{\gamma}_{n}^{i}-\left[m \mathbf{e}+(1-m) \mathbf{e}^{e p}\right]^{T} \Delta \mathbf{E}_{n}^{i}, \\
& \mathbf{D}_{n+1}^{i+1}=\mathbf{D}_{n}+\left[m \mathbf{e}+(1-m) \mathbf{e}^{e p}\right] \Delta \boldsymbol{\gamma}_{n}^{i}+\left[m \boldsymbol{\epsilon}+(1-m) \boldsymbol{\epsilon}^{e p}\right] \Delta \mathbf{E}_{n}^{i}
\end{aligned}
$$

where

$$
m=\frac{D_{c}-\mathbf{n}_{o}^{T} \mathbf{D}_{n}}{\mathbf{n}_{o}^{T}\left(\mathbf{D}_{n+1}^{i}-\mathbf{D}_{n}\right)} .
$$

$\underline{\text { Case } 3}$ If $\left(\mathbf{D}_{n}-\mathbf{D}_{o}\right)^{T} \mathbf{n}_{o}=D_{c}$, the material has already been electrically saturated at load $\mathbf{f}_{n}$, thus

$$
\boldsymbol{\tau}_{n+1}^{i+1}=\boldsymbol{\tau}_{n}+\mathbf{c}^{e p} \Delta \boldsymbol{\gamma}_{n}^{i}-\left(\mathbf{e}^{e p}\right)^{T} \Delta \mathbf{E}_{n}^{i}, \mathbf{D}_{n+1}^{i+1}=\mathbf{D}_{n}+\mathbf{e}^{e p} \Delta \boldsymbol{\gamma}_{n}^{i}+\boldsymbol{\epsilon}^{e p} \Delta \mathbf{E}_{n}^{i}
$$


The generalized internal and residual forces required for the next iterative are :

$$
\left\{\begin{array}{l}
\mathbf{P}_{m}\left(\mathbf{q}_{n+1}^{i+1}\right) \\
\mathbf{P}_{e}\left(\mathbf{q}_{n+1}^{i+1}\right)
\end{array}\right\}=\left\{\begin{array}{c}
\int_{\Omega} \mathbf{B}_{m}^{T} \boldsymbol{\tau}_{n+1}^{i+1} d \Omega \\
\int_{\Omega} \mathbf{B}_{e}^{T} \mathbf{D}_{n+1}^{i+1} d \Omega
\end{array}\right\},\left\{\begin{array}{l}
\boldsymbol{\psi}_{m}\left(\mathbf{q}_{n+1}^{i+1}\right) \\
\boldsymbol{\psi}_{e}\left(\mathbf{q}_{n+1}^{i+1}\right)
\end{array}\right\}=\left\{\begin{array}{l}
\mathbf{P}_{m}\left(\mathbf{q}_{n+1}^{i+1}\right) \\
\mathbf{P}_{e}\left(\mathbf{q}_{n+1}^{i+1}\right)
\end{array}\right\}-\mathbf{f}_{n+1}
$$

The convergence criterion is taken to be the simultaneous satisfaction of :

$$
\left.\left|\boldsymbol{\psi}_{m}\right|<\text { tolerance (if }\left|\mathbf{f}_{m}\right|=0\right), \quad\left|\boldsymbol{\psi}_{m}\right| /\left|\mathbf{f}_{m}\right|<\text { tolerance (if }\left|\mathbf{f}_{m}\right| \neq 0 \text { ) }
$$

and

$$
\left.\left|\boldsymbol{\psi}_{e}\right|<\text { tolerance (if }\left|\mathbf{f}_{e}\right|=0\right), \quad\left|\boldsymbol{\psi}_{e}\right| /\left|\mathbf{f}_{e}\right|<\text { tolerance (if }\left|\mathbf{f}_{e}\right| \neq 0 \text { ). }
$$

Polarization switching is first checked due to the inherent difficulty of handling the instantaneous polarization switching in incremental method. As $\mathbf{c}^{e p}, \mathbf{e}^{e p}$ and $\boldsymbol{\epsilon}^{e p}$ are constant matrices, $\boldsymbol{\tau}_{n+1}^{i+1}$ and $\mathbf{D}_{n+1}^{i+1}$ can be obtained by Eqn.(26) without partitioning $\Delta \boldsymbol{\gamma}_{n}^{i}$ and $\Delta \mathbf{E}_{n}^{i}$ into smaller increments.

\section{NUMERICAL INVESTIGTION}

The above constitutive model has been implemented using the standard four-node quadrilateral element. The element contains four nodes and the nodal d.o.f.s include the electric potential as well as the displacements along the $x_{1}$ - and $x_{3}$-axes. The material being considered is PZT-4 whose material coefficients are given in Table 1 . The reported coercive electric field is $1 \mathrm{MV} / \mathrm{m}$ [39-41] from which and from the traction-free conditions, the critical electric displacement $D_{\mathrm{c}}$ is calculated to be $0.010 \mathrm{C} / \mathrm{m}^{2}$. The remnant polarization $D_{\mathrm{o}}$ is taken to be $0.2 \mathrm{C} / \mathrm{m}^{2}$ [6]. However, the computed J-integrals are independent of $D_{\mathrm{o}}$ unless polarization switching occurs.

\begin{tabular}{|c|c|c|c|c|c|c|c|c|c|c|c|}
\hline & $c_{11}$ & $c_{12}$ & $c_{13}$ & $c_{33}$ & $c_{44}$ & $e_{31}$ & $e_{33}$ & $e_{15}$ & $\epsilon_{11}$ & $\epsilon_{33}$ & $D_{c} *$ \\
\hline PZT-4 & 13.9 & 7.78 & 7.743 & 11.3 & 2.56 & -6.98 & 13.84 & 13.44 & 6.00 & 5.47 & 0.010 \\
\hline
\end{tabular}

Table 1. Material properties for PZT-4 [11]. $c_{i j}$ 's, $e_{i j}$ 's, $\in_{i j}$ 's and $D_{c}$ are expressed in terms of $\mathrm{GPa}, \mathrm{C} / \mathrm{m}^{2}, 10^{-9} \mathrm{C} / \mathrm{Vm}$ and $\mathrm{C} / \mathrm{m}^{2}$, respectively.

5.1 J-Integral for Piezoelectric Materials - The $J$-integral for piezoelectric materials was derived by Pak [5] as :

$$
J=\int_{\Gamma}\left(h n_{1}-\tau_{i j} n_{j} u_{i, 1}+D_{i} n_{i} E_{1}\right) d s
$$

where

$h$ is the electric enthalpy that satisfies $\partial h / \partial \gamma_{i j}=\tau_{i j}$ and $\partial h / \partial E_{i}=-D_{i}$, 
$n_{i}$ is the unit outward normal component along path $\Gamma$.

Provided that the end points of $\Gamma$ are taken at the two crack faces, the crack faces are traction-free and the crack is impermeable, the integral is path-independent and equivalent to the energy release rate for linear piezoelectric materials [5].

For the present "elastoplastic" piezoelectric material model, the electric enthalpy under monotonic loading can be taken as :

$$
h=\int_{\text {initial }}^{\text {current }}\left\{\begin{array}{c}
\boldsymbol{\tau} \\
\mathbf{D}
\end{array}\right\}^{T}\left\{\begin{array}{c}
d \boldsymbol{\gamma} \\
-d \mathbf{E}
\end{array}\right\}=\int_{\text {initial }}^{\text {current }}\left\{\begin{array}{c}
\boldsymbol{\tau} \\
\mathbf{D}
\end{array}\right\}^{T}\left\{\begin{array}{c}
d \boldsymbol{\gamma} \\
-d \mathbf{E}^{e}
\end{array}\right\}+\int_{\text {initial }}^{\text {current }}\left\{\begin{array}{c}
\boldsymbol{\tau} \\
\mathbf{D}
\end{array}\right\}^{T}\left\{\begin{array}{c}
\mathbf{0} \\
-d \mathbf{E}^{p}
\end{array}\right\}
$$

where initial and current refer to the initial configuration $\left(\boldsymbol{\gamma}=\mathbf{E}^{e}=\mathbf{E}^{p}=0\right)$ and the current configuration. By invoking the linear piezoelectric relation in Eqn.(6),

$$
h=\int_{\text {initial }}^{\text {current }}\left\{\begin{array}{c}
\boldsymbol{\gamma} \\
-\mathbf{E}^{e}
\end{array}\right\}^{T}\left[\begin{array}{cc}
\mathbf{c} & \mathbf{e}^{T} \\
\mathbf{e} & -\mathbf{\epsilon}
\end{array}\right]+\left\{\begin{array}{c}
\mathbf{0} \\
\mathbf{D}
\end{array}\right\}^{T}\left\{\begin{array}{c}
d \boldsymbol{\gamma} \\
-d \mathbf{E}^{e}
\end{array}\right\}-\int_{\text {initial }}^{\text {current }} \mathbf{D}^{T} d \mathbf{E}^{p}=\frac{1}{2}\left\{\begin{array}{c}
\boldsymbol{\tau} \\
\mathbf{D}+\mathbf{D}_{o}
\end{array}\right\}^{T}\left\{\begin{array}{c}
\boldsymbol{\gamma} \\
-\mathbf{E}^{e}
\end{array}\right\}-\int_{0}^{\mathbf{E}^{p}} \mathbf{D}^{T} d \mathbf{E}^{p}
$$

For non-zero $\mathbf{E}^{p}$, the first component of $\mathbf{E}^{p}$ is always zero and $D_{3}$ is equal to $D_{o}+D_{c}$ and $-D_{o}-D_{c}$ when the poling direction is respectively parallel and anti-parallel to $x_{3}$. Thus,

$$
h=\frac{1}{2}\left[\boldsymbol{\tau}^{T} \boldsymbol{\gamma}-\left(\mathbf{D}+\mathbf{D}_{o}\right)^{T}\left(\mathbf{E}-\mathbf{E}^{P}\right)\right]-\int_{0}^{E_{3}^{P}} D_{3} E_{3}^{P}=\frac{1}{2}\left[\boldsymbol{\tau}^{T} \boldsymbol{\gamma}-\left(\mathbf{D}+\mathbf{D}_{o}\right)^{T}\left(\mathbf{E}-\mathbf{E}^{P}\right)\right]-D_{3} E_{3}^{P} .
$$

5.2 Linear Fracture Analysis - The centrally cracked plane strain square panel in Fig.3 is considered and $w / a$ is taken to be 20 which aims at simulating the crack-in-infinite-plate configuration. The poling direction is the positive $x_{3}$-direction. Owing to symmetry, a quarter of the panel is modelled. Fig.4 shows the finite element mesh which includes 1424 elements and 1515 nodes. To capture the stress and electric displacement concentrations at the crack tip, a very high mesh density is employed as seen in Fig.5. The side length of the elements at the crack tip is around $10^{-5} \mathrm{a}$. The crack surfaces are taken to be impermeable and traction-free. The present study aims at checking whether the mesh is dense enough to obtain consistent and path independent $J$-integrals. For different combinations of electric and mechanical loads, $J$-integrals are computed along the six contours depicted in Fig.5 and listed in Table 2. All the contours pass through the second order Gaussian points of the elements. $\Gamma_{1}$ to $\Gamma_{4}$ are approximately at a distance $10^{-4} a$ from the crack tip whereas $\Gamma_{5}$ and $\Gamma_{6}$ are approximately at a distance $a$ from the crack tip. The J-integrals along the contours closer to and farther away from the crack tip are respectively marginally higher and lower than the exact energy release rate $[14,19]$ whereas the largest difference is around $3 \%$ only. As a 
matter of fact, J-integrals have been computed along more than ten different paths between $\Gamma_{1}$ and $\Gamma_{6}$. For the same electromechanical loading, all paths yield close results. Previous investigations have obtained the following energy release rate for centrally cracked infinite PZT-4 plate $[14,19]$ :

$$
G_{\text {linear }}=10^{-9} a\left(0.0363 \sigma_{\infty}^{2}+0.0373 \sigma_{\infty} E_{\infty}-13.83 E_{\infty}^{2}\right) .
$$

Using the least square fit, the expression below is obtained for the average integrals computed along $\Gamma_{5}$ and $\Gamma_{6}$ :

$$
J_{\text {linear }}=10^{-9} a\left(0.0360 \sigma_{\infty}^{2}+0.0370 \sigma_{\infty} E_{\infty}-13.78 E_{\infty}^{2}\right)
$$

which agrees closely with $G_{\text {linear }}$. The mesh density is considered to be adequate.

\begin{tabular}{|c|c|c|c|c|c|c|c|c|}
\hline \multicolumn{2}{|c|}{ Load Case } & \multirow[t]{2}{*}{$\Gamma_{1}$} & \multirow[t]{2}{*}{$\Gamma_{2}$} & \multirow[t]{2}{*}{$\Gamma_{3}$} & \multirow[t]{2}{*}{$\Gamma_{4}$} & \multirow[t]{2}{*}{$\Gamma_{5}$} & \multirow[t]{2}{*}{$\Gamma_{6}$} & \multirow{2}{*}{$\begin{array}{c}\text { Exact } \\
{[14]}\end{array}$} \\
\hline$\sigma_{\infty}(\mathrm{MPa})$ & $E_{\infty}(100 \mathrm{kV} / \mathrm{m})$ & & & & & & & \\
\hline 0.0 & 1.0 & -142.1 & -142.0 & -142.0 & -142.0 & -137.7 & -137.8 & -138.3 \\
\hline 0.0 & -1.0 & -142.1 & -142.0 & -142.1 & -142.0 & -137.7 & -137.8 & -138.3 \\
\hline 1.0 & 0.0 & 37.33 & 37.34 & 37.34 & 37.35 & 36.12 & 35.93 & 36.30 \\
\hline 1.0 & 1.0 & -100.9 & -100.9 & -100.8 & -100.8 & -97.89 & -98.10 & -98.27 \\
\hline 1.0 & -1.0 & -108.6 & -108.6 & -108.6 & -108.6 & -105.3 & -105.6 & -105.7 \\
\hline 1.0 & 0.05 & 37.17 & 37.17 & 37.18 & 37.19 & 35.96 & 35.77 & 36.14 \\
\hline 1.0 & -0.05 & 36.79 & 36.79 & 36.80 & 36.80 & 35.59 & 35.40 & 35.77 \\
\hline 1.0 & 0.1 & 36.30 & 36.30 & 36.31 & 36.32 & 35.11 & 34.93 & 35.29 \\
\hline 1.0 & -0.1 & 35.53 & 35.53 & 35.54 & 35.54 & 34.37 & 34.18 & 34.54 \\
\hline 0.1 & 1.0 & -141.3 & -141.3 & -141.3 & -141.3 & -137.0 & -137.0 & -137.6 \\
\hline 0.1 & -1.0 & -142.1 & -142.1 & -142.0 & -142.0 & -137.8 & -137.7 & -138.3 \\
\hline 0.5 & 1.0 & -130.8 & -130.8 & -130.8 & -130.7 & -126.8 & -126.9 & -127.4 \\
\hline 0.5 & -1.0 & -134.6 & -134.6 & -134.6 & -134.6 & -130.5 & -130.6 & -131.1 \\
\hline
\end{tabular}

Table 2. Comparison of $J$-integrals for linear PZT-4, see Fig.6a

5.3 Nonlinear Fracture Analysis : Crack Perpendicular to the Poling Axis - In the present and next subsections, the following nonlinear models are considered :

- switching model in which $D_{\mathrm{o}}=0.2 \mathrm{C} / \mathrm{m}^{2}$ and $D_{\mathrm{c}}=0.010 \mathrm{C} / \mathrm{m}^{2}$

- non-switching model in which $D_{\mathrm{o}}=0$ and $D_{\mathrm{c}}=0.010 \mathrm{C} / \mathrm{m}^{2}$

For the setup in Fig.3a, switching can be triggered by negative $E_{\infty}$ and negative $\sigma_{\infty}$. In the present study, it will first be assumed that a mode I crack with $a=1 \mathrm{~cm}$ starts to propagate at $\sigma_{\infty}=2 \mathrm{MPa}$ and $E_{\infty}=0$ [14]. The computed J-integrals along the six contours in Fig.5 are listed in Table 3. As switching is not triggered, both the switching and non-switching models yield the same J-integral. The four local J-integrals computed along $\Gamma_{1}$ to $\Gamma_{4}$ which are very close to the crack tip and cross the electric saturation zone are essentially consistent and their mean is $1.521 \mathrm{~N} / \mathrm{m}$. Unlike the linear predictions in the last subsection, the two global J-integrals computed along $\Gamma_{5}$ to $\Gamma_{6}$ which are 
well away from the electric saturation zone are different from the local J-integrals.

Table 3. J-integrals and energy release rates (in N/m) at $\sigma_{\infty}=2 \mathrm{MPa}$ and $E_{\infty}=0$, see Fig.6a

\begin{tabular}{|c|c|c|c|c|c|c|c|}
\hline \multicolumn{4}{|c|}{ local J-integral $(\mathrm{N} / \mathrm{m})$} & \multicolumn{3}{c|}{ global J-integral $(\mathrm{N} / \mathrm{m})$} & \multicolumn{2}{c|}{ energy release rate $(\mathrm{N} / \mathrm{m})$} \\
\hline$\Gamma_{1}$ & $\Gamma_{2}$ & $\Gamma_{3}$ & $\Gamma_{4}$ & $\Gamma_{5}$ & $\Gamma_{6}$ & local & global \\
\hline 1.519 & 1.522 & 1.522 & 1.521 & 1.444 & 1.437 & 1.880 & 1.452 \\
\hline
\end{tabular}

Table 3 also lists the global (or apparent) and local energy release rate derived by Gao et al based on their electric saturation strip model $[13,14]$. The latter is :

$$
G_{\text {local }}=10^{-9} a\left(0.047 \sigma_{\infty}^{2}+0.910 \sigma_{\infty} E_{\infty}+4.43 E_{\infty}^{2}\right) \text {. }
$$

The global energy release rate and the global $J$-integral are obtained by considering integration paths not crossing the saturation strip and saturation zone, respectively. Along these paths, the field variables are not sensitive to the electric ductility. Hence, the global energy release rate, the global $J$-integral rate and the linear energy release rate are close to each other. Zhang et al [19] and Han et al [43] argued that the electric saturation zone size or strip length in the saturation strip model remains unchanged during crack propagation and thus only the mechanical energy is taking into account in the local energy release rate. The local energy release rate provides a physical basis for the mechanical energy release rate. In this context, the observation that the computed local $J$ integrals are smaller than the local energy release rate can readily be understood as the electric contribution in the $J$-integrals are always negative.

By adopting the local J-integral as the fracture criterion and taking $1.521 \mathrm{~N} / \mathrm{m}$ as its critical value for cracking, the relation between the fracture stress and the electric field is obtained by trial and error. Fig. 6 shows the variations of the fracture stress with respect to the applied electric field when the linear or global energy release rate $G_{\text {linear }}$, the local energy release rate $G_{\text {local }}$, the local $J$ integral using the switching model $J_{\text {local }}$ (switching) and the local J-integral using the nonswitching model $J_{\text {local }}$ (non-switching) are used as the fracture criteria. It can be seen that the fracture stresses predicted by $G_{\text {local }}$ and $J_{\text {local }}$ (non-switching) drop monotonically as the electric field increases. This agrees with the experimental observation that positive and negative electric fields promote and impede crack propagation, respectively. Within the $E_{\infty}$ range shown, the fracture stress based upon $G_{\text {local }}$ varies with $E_{\infty}$ roughly in a linear manner and intercepts with the $E_{\infty}$-axis whereas the fracture stress based upon the $J_{\text {local }}$ does not. Qualitatively, the latter stress agrees better with the experimental data measured by Park and Sun [11] using compact tension specimens in the sense that crack propagation must be assisted by tensile stress even if the electric 
field is very large.

In comparing the fracture stresses predicted by $J_{\text {local }}$ (switching) and $J_{\text {local }}$ (non-switching), three ranges of the electric field can be considered. For $E_{\infty}>-75 \mathrm{kV} / \mathrm{m}$, the electric displacement along the $x_{3}$-direction inside the panel is predominately positive and switching does not occur. The two fracture stresses are identical. For $-75 \mathrm{kV} / \mathrm{m}>E_{\infty}>-174 \mathrm{kV} / \mathrm{m}$, elements at the crack tip vicinity are switched and the two fracture stresses deviate. For $E_{\infty}<-174 \mathrm{kV} / \mathrm{m}$, a considerable portion of the elements at the crack tip vicinity are switched and become poled in the negative $x_{3}$-direction. It is therefore natural that the fracture stress drops when a strong negative $E_{\infty}$ is applied as the latter is along the current poling direction of most elements at the crack tip region. The observation is physically the same as the one when positive $E_{\infty}$ is applied to the panel which is poled along the positive $x_{3}$-direction.

To supplement the above paragraph, Fig.7a to Fig.7f are prepared to portray the predicted electric saturation zones by the switching model at the thresholds of cracking for $E_{\infty}$ equal to -175 , $-174,-100,0,10$ and $200 \mathrm{kV} / \mathrm{m}$, the lengths of the saturation zones are respectively $0.0056 a$, $0.0033 a, 0.0009 a, 0.0025 a, 0.021 a$ and $0.06 a$. By realizing that both positive $\sigma_{\infty}$ and positive $E_{\infty}$

induce a positive saturated zone within which $D_{3}$ equals $D_{o}+D_{c}$ whereas negative $E_{\infty}$ induces a negative saturated zone within which $D_{3}$ equals $-D_{o}-D_{c}$, the aforementioned lengths can be readily understood. The saturation zone is positive and increases monotonically from $E_{\infty}=0$ to $200 \mathrm{kV} / \mathrm{m}$. From $E_{\infty}=0$ to $-75 \mathrm{kV} / \mathrm{m}$, the saturation zone is still positive but diminishes in size. Fig.7c shows the small and negative saturation zone at $E_{\infty}=-100 \mathrm{kV} / \mathrm{m}$. Stronger negative $E_{\infty}$ results in progressively larger negative saturation zone as noted in Fig.7e ad Fig.7g. It can also be noted that geometry of the present saturation zones is considered different from that of the saturation strip assumed by Gao et al $[13,14]$.

5.4 Nonlinear Fracture Analysis : Crack Parallel to the Poling Axis - This study is depicted in Fig. $3 b$. The panel is poled and the far-field electric field is applied along the positive $x_{1}$-direction. The crack tip stress and electric displacement concentrations are induced solely by $\sigma_{\infty}$ whereas $E_{\infty}$ does not contribute to the concentrations. The global and local J-integrals are dominated by $\sigma_{\infty}$ and insensitive to $E_{\infty}$. Table 4 lists the computed J-integrals for different $E_{\infty}$ along the six contours in Fig.5 by fixing $\sigma_{\infty}$ at $2.302 \mathrm{MPa}$. The global and local J-integrals are different as electric saturation is induced by the applied $\sigma_{\infty}$. There is no practical difference between the predictions yielded by the switching and non-switching models as the applied negative $E_{\infty}$ is far from 
sufficient to trigger switching whereas the applied $\sigma_{\infty}$ may induce $90^{\circ}$ switching but not the $180^{\mathrm{O}}$ switching. The former, however, is not considered in the present constitutive model. Table 4 shows that all the local J-integrals computed at $\sigma_{\infty}=2.302 \mathrm{MPa}$ are very close to $1.521 \mathrm{~N} / \mathrm{m}$. If the value is adopted as the critical one for crack propagation, one can see that the fracture stresses are insensitive to the electric field and close to $2.302 \mathrm{MPa}$. The conclusion agrees with that in reference [13] which does not take into account of the electric saturation.

Table 4. Different J-integrals computed at $\sigma_{\infty}=2.302 \mathrm{MPa}$ and different $E_{\infty}$, see Fig.5b.

\begin{tabular}{|c|c|c|c|c|c|c|c|}
\hline \multirow{2}{*}{$E_{\infty}(100 \mathrm{kV} / \mathrm{m})$} & \multicolumn{5}{|c|}{ local J-integral $(\mathrm{N} / \mathrm{m})$} & \multicolumn{2}{c|}{ global J-integral $(\mathrm{N} / \mathrm{m})$} \\
\cline { 2 - 7 } & $\Gamma_{1}$ & $\Gamma_{2}$ & $\Gamma_{3}$ & $\Gamma_{4}$ & mean & $\Gamma_{5}$ & $\Gamma_{6}$ \\
\hline-2 & 1.520 & 1.519 & 1.518 & 1.518 & 1.519 & 1.468 & 1.458 \\
\hline-1 & 1.521 & 1.520 & 1.519 & 1.518 & 1.520 & 1.468 & 1.458 \\
\hline 0 & 1.523 & 1.522 & 1.521 & 1.520 & 1.522 & 1.468 & 1.458 \\
\hline 1 & 1.525 & 1.524 & 1.523 & 1.522 & 1.524 & 1.468 & 1.458 \\
\hline 2 & 1.525 & 1.525 & 1.523 & 1.522 & 1.524 & 1.468 & 1.458 \\
\hline
\end{tabular}

\section{CLOSURE}

A simple nonlinear constitutive model for piezoelectric ceramics that accounts for electric saturation and $180^{\circ}$ domain switching is devised. Compared to others, the present model can be conveniently implemented in finite element computation. The pertinent incremental iterative procedures are formulated and used to study the fracture behaviour of mode I crack.

The global J-integrals which are computed along the contours well away from the electric saturation zone remain virtually unchanged with respect to the linear solution. Nevertheless, the local J-integrals which are computed along the contours close to the crack-tip and within the electric saturation zone are sensitive to the constitutive non-linearity. Analogous to the local energy release rate in the saturation strip model proposed by Gao and his coworkers [12-14], the local $J$ integral is here postulated as the fracture criterion and used to study the propagation behaviour of a crack with half-length equal to $1 \mathrm{~cm}$ inside a PTZ-4 panel. Two setups are considered.

In the first setup, the poling direction, remote stress and remote electric field are perpendicular to the crack. When the electric field is positive (along the poling direction), crack propagation is promoted and the fracture stress becomes smaller. This is in qualitative agreement with the experimental results, the predictions based on the mechanical energy release rate [11] and the local 
energy release rate in the electric saturation strip model [12-14]. The present finite element computation provides a more realistic account of the electric saturation compared to the electric saturation strip model whose saturation zone is a geometric line (zero thickness). For the predicted fracture stresses, the present one is qualitatively more consistent to experimental results obtained by Park and Sun using compact tension specimens [11] in the sense that the fracture stress decreases gradually as the positive electric field increases but does not drop to zero even for a very strong electric field. When the electric field is negative, polarization switching can occur around the crack tip. For strong and negative electric fields, a considerable amount of the material will be switched at the crack tip region. The negative electric field is then parallel to and along the new poling direction of the switched material. This again leads to a smaller fracture stress and promotes crack propagation. Unfortunately, there has not been any experimental evidence to support this point.

In the second setup, the far-field stress are perpendicular to the poling direction, far-field electric field and the crack. Electric saturation is induced by the stress concentration effect of the crack and electromechanical coupling effect of the material. For the considered range of electric field, the fracture stress predicted by the local J-integral is practically constant and is not affected by the electric field. This is in agreement with the conclusion by Gao and his coworkers [13].

Acknowledgment - The financial support of the Research Grant Council of Hong Kong in the form of a CERG Grant (project no. HKU 7083/00E) is gratefully acknowledged. 


\section{REFERENCES}

1. B.Jaffe, W.R.Cook and H.Jaffe, Piezoelectric Ceramics, Academic Press, London, 1971

2. V.Z.Parton, "Fracture mechanics of piezoelectric materials", Acta Astronaut., 3, 671-683 (1976)

3. W.F.J.Deeg, The Analysis of Dislocation, Crack and Inclusion Problems in Piezoelectric Solids, Ph.D. Thesis, Stanford University, 1980

4. R.M.McMeeking, "Electrostrictive stresses near crack-like flaw", Journal of Applied Mathematics \& Physics, 40, 615-627 (1989)

5. Y.E.Pak, "Crack extension force in a piezoelectric materials", J.Appl.Mech., 57, 647-653 (1990)

6. Y.E.Pak, "Linear electro-elastic fracture mechanics of piezoelectric materials", Int.J.Fracture, 54, 79-100 (1992)

7. H.Sosa, "On the fracture mechanics of piezoelectric solids", Int.J.Solids Structures, 29, 2613$2622(1992)$

8. Z.Suo, C.M.Huo, D.M.Barnett and J.R.Willis, "Fracture mechanics for piezoelectric ceramics", J.Mech.Phys.Solids, 41, 1155-1176 (1992)

9. H.C.Cao and A.G.Evans, "Electric-field-induced fatigue crack growth in piezoelectrics", J.Amer.Ceram.Soc., 77, 1783-1786 (1994)

10. M.L.Dunn, "The effects of crack face boundary conditions on the fracture mechanics of piezoelectric solids", Engineering Fracture Mechanics, 48, 25-39 (1994)

11. S.Park and C.T.Sun, "Fracture criteria for piezoelectric ceramics", J.Amer.Ceram.Soc., 78, 1475-1480 (1995)

12. H.Gao and D.M.Barnett, "An invariance property of local energy relaese rates in a strip saturation model of piezoelectric fracture", Int.J.Fracture, 79, R25-R29 (1996)

13. H.Gao, T.Y.Zhang ang P.Tong, "Local and global energy release rates for an electrically yielded crack in a piezoelectric ceramic", J.Mech.Phys.Solids, 45, 491-510 (1997)

14. C.C.Fulton and H.Gao, "Electrical nonlinearity in fracture of piezoelectric ceramics", Appl. Mech.Reviews, 50, S56-S63 (1997)

15. S.Kumar and R.N.Singh, "Energy release rate and crack propagation in piezoelectric materials. Part I: Mechanical/electrical load", Acta Mater., 45, 849-857 (1997)

16. S.Kumar and R.N.Singh, "Energy release rate and crack propagation in piezoelectric materials. Part II: Combined mechanical and electrical loads", Acta Mater., 45, 859-868 (1997)

17. S.Kumar and R.N.Singh, "Effect of the mechanical boundary condition at the crack surfaces on the stress distribution at the crack tip in piezoelectric materials", Materials Science \& Engineering A, 252, 64-77 (1998)

18. T.Y.Zhang and P.Tong, "Fracture mechanics for a mode III crack in a piezoelectric material", Int.J.solids Struct., 33, 343-359 (1996)

19. T.Y.Zhang, C.F.Qian and P.Tong, "Linear electro-elastic analysis of a cavity or a crack in piezoelectric material", Int.J.Solids Structures, 35, 2121-2149 (1998)

20. W.Yang and T.Zhu, "Switch-toughening of ferroelectrics subjected to electric fields", J.Mech.Phys.Solids, 46, 291-311 (1998)

21. T.Zhu and W.Yang, "Fatigue crack growth in ferroelectrics driven by cyclic electric loading", J.Mech.Phys.Solids, 47, 81-97 (1999)

22. K.H.Chan and N.W.Hagood, "Modeling of nonlinear piezoceramics for structural actuation", 
Smart Structures and Materials 1994: Smart Structures and Intelligent Systems, Ed. N.W. Nagood, Proc. SPIE, 2190, 194-205 (1994)

23. S.C.Hwang, C.S.Lynch and R.M.McMeeking, "Ferroelectric/ferroelastic interactions and a polarization switching model”, Acta Metall.Mater., 43, 2073 (1995)

24. S.C.Hwang, J.E.Huber, R.M.McMeeking and N.A.Fleck, "The simulation of switching in polycrystalline ferroelectric ceramics", Journal of Applied Physics, 84, 1530-1540 (1998)

25. S.C.Hwang and R.M.McMeeking, "A finite element model of ferroelastic polycrystals", Int.J.Solids Structures, 36, 1541-1556 (1999)

26. Y.Fotinich and G.P.Carman, "Modeling of polarization switching in piezoceramics", Smart Structures and Materials 1998: Mathematics and Control in Smart Structures, Ed. V.V.Varadan, Proc. SPIE, 3323, 96-108 (1998)

27. R.J.Chen and P.S.Peercy, "Three dimensional dynamic electromechanical constitutive relations for ferroelectric materials", Int.J.Solids Struct., 16, 1059-1067 (1980)

28. P.J.Chen and M.M.Marsden, "One dimensional polar responses of the electrooptic ceramic PLZT 7/65/35 due to domain switching”, Acta Mech., 41, 255-264(1981)

29. A.R.Freeman and S.P.Joshi, "Numerical modeling of PZT nonlinear electromechanical behavior", Smart Structures and Materials 1996: Mathematics and Controls in Smart Structures, Ed. V.V.Varadan and J.Chandra, Proc. SPIE, 2715, 602-613 (1996)

30. D.Hughes and J.T.Wen, "Preisach modeling of piezoceramic and shape memory alloy hysteresis", Smart Mater.Struct., 6, 287-300 (1997)

31. M.Kamlah and C.Tsakmakis, "Phenomenological modeling of the nonlinear electro-mechanical coupling in ferroelectrics", Int.J.Solids Struct., 36, 669-695 (1999)

32. E.Bassiouny, G.A. Maugin, "Thermodynamic formulation for coupled electromagenetic hysteresis effects - IV. Combined electromechanical loading”, Int.J.Engng.Sci., 27, 989-1000 (1989)

33. S.P.Joshi, "Nonlinear constitutive relations for piezoelectric materials", Smart Mater.Struct., 1, 80-83(1992)

34. C.L.Hom, N.Shankar, "A fully coupled constitutive model for electostrictive ceramic materials", J.Intelligent Material Systems \& Structures, 5, 795-801 (1994)

35. K.Ghandi, Nonlinear Modeling and Characterization Techniques for Phase Transitions in Electro-Mechanically Coupled Devices, Ph.D. Thesis, Massachusetts Institute of Technology, Cambridge, MA, 1998

36. X.Chen, D.N.Fang, K.C.Hwang, "A mesoscopic model of the constitutive behavior of monocrystalline ferroelectrics", Smart Mater.Struct., 6, 145-151 (1997)

37. S.J.Kim, "A simple continuum model for polarization reversals in ferroelectric", Smart Mater. Struct., 7, 572-579 (1998)

38. X.Gong, Z.Suo, "Reliability of ceramic multilayer actuators: a nonlinear finite element simulation", J.Mech.Phys.Solids, 44, 751-769 (1996)

39. S.S.Park, G.P.Carman, S.B.Park, "Moire interferometer applied to a piezoceramic containing a simulated void”, Smart Structures and Materials 1997: Smart Materials Technologies, Ed. W.C.Simmons, I.A.Aksay and D.R.Huston, Proc. SPIE, 3040, 120-128 (1997)

40. S.B.Park, S.S.Park, H.T.Carman, "Measuring strain distribution during mesoscopic domain reorientation in a ferroelectric material", Journal of Engineering Materials and Technology, 120, $1-7(1998)$ 
41. D.Wang, Y.Fotinich, G.P.Carman, "Influence of temperature on the electromechanical and fatigue behavior of piezoelectric ceramics", Journal of Applied Physics, 83, 5342-5350 (1998)

42. K.M.Ghandi, N.W.Hagood, "Nonlinear finite element modeling of phase transitions in electromechanically coupled material", Smart Structures and Materials 1996 : Mathematics and Control in Smart Structures, Ed. V.V.Varadan \& J.Chandra, Proc. SPIE, 2715, 121-140, (1996)

43. X.-L.Han, Z.-C.Wang, "Interacting multiple cracks in piezoelectric materials", Int.J.Solids Struct., 36, 4183-4202 (1999) 


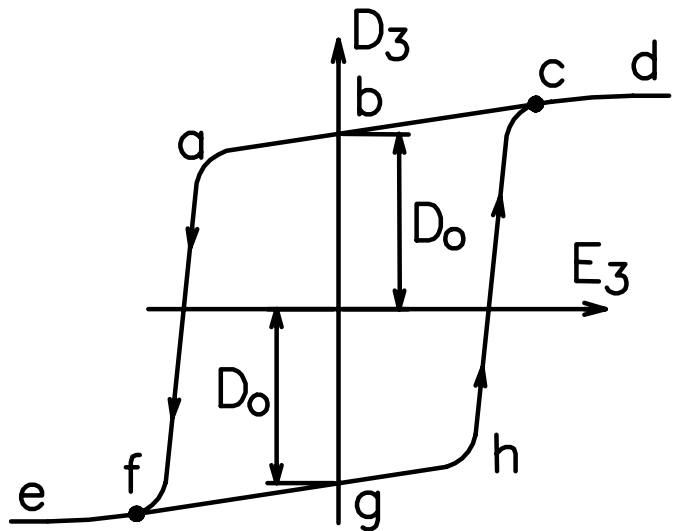

(a)

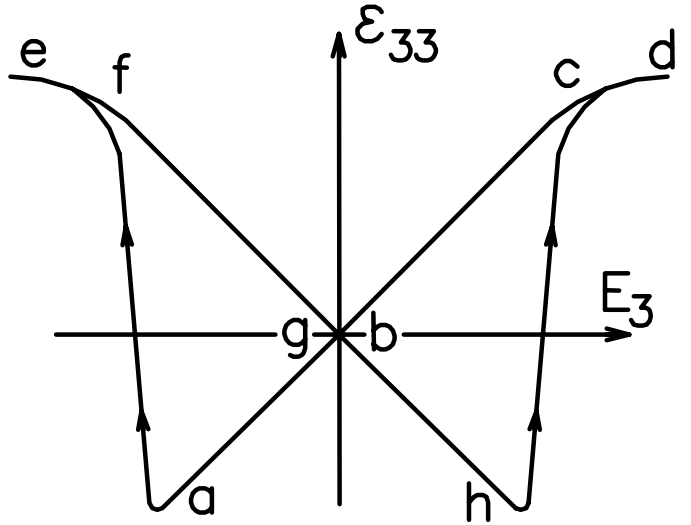

(b)

Fig.1 Typical (a) electric displacement-electric field and (b) strain-electric field relations for a piezoelectric crystal poled along the y-direction and under zero stress. The arrows indicate that the associated switching paths are irreversible.

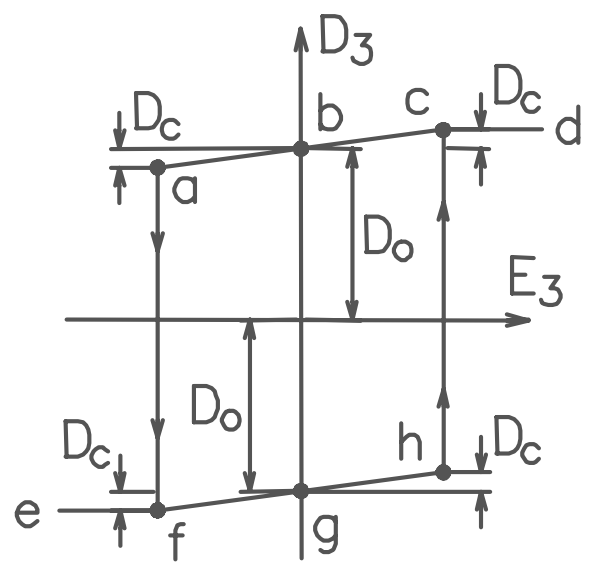

(a)

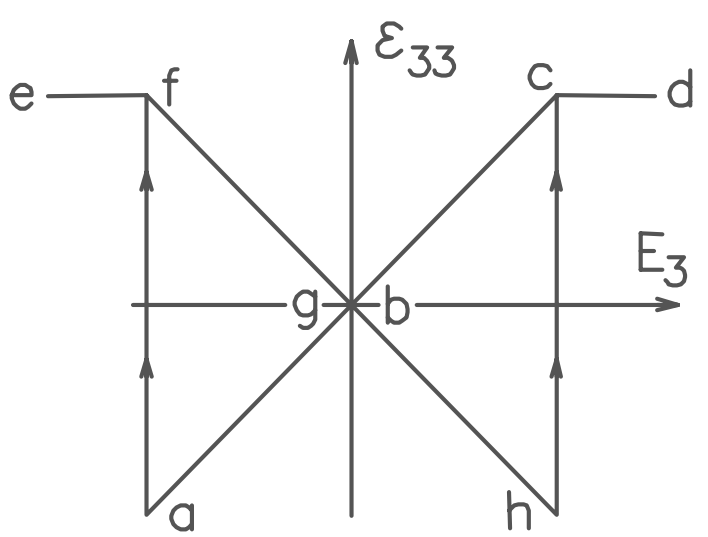

(b)

Fig.2 The electric displacement-electric field and (b) strain-electric field relations of the present material model for domain switching and electric saturation 


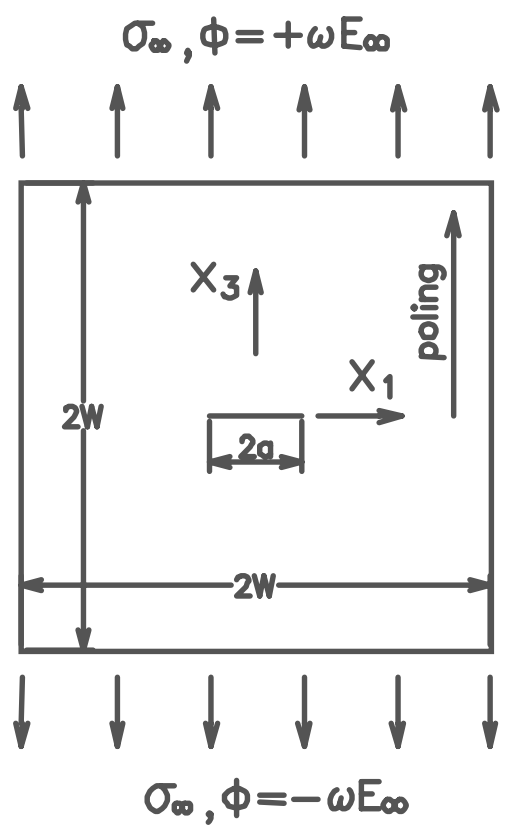

(a)

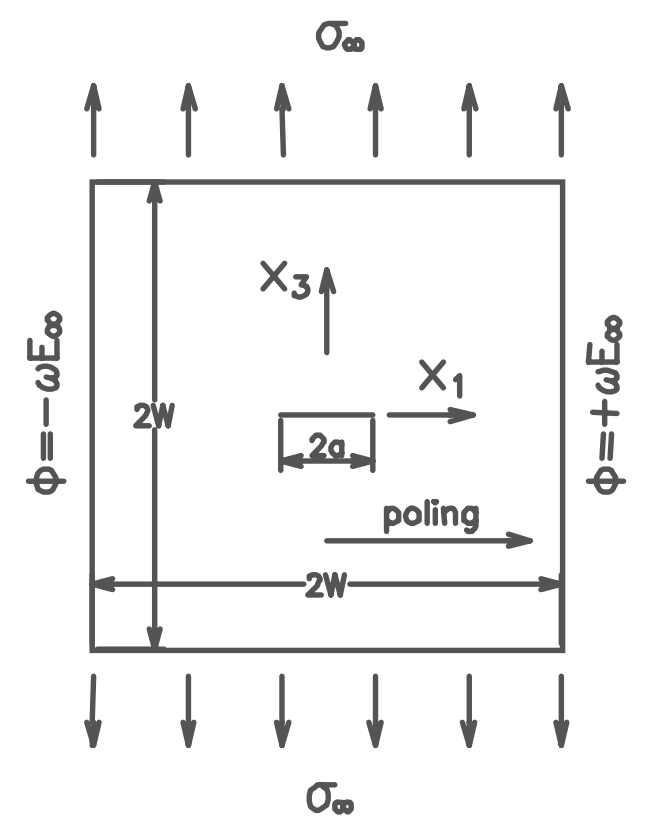

(b)

Fig.3. Centrally cracked panels with (a) the poling direction and electric field perpendicular to the crack and (b) the poling direction and electric field parallel to the crack. $w / a=20$

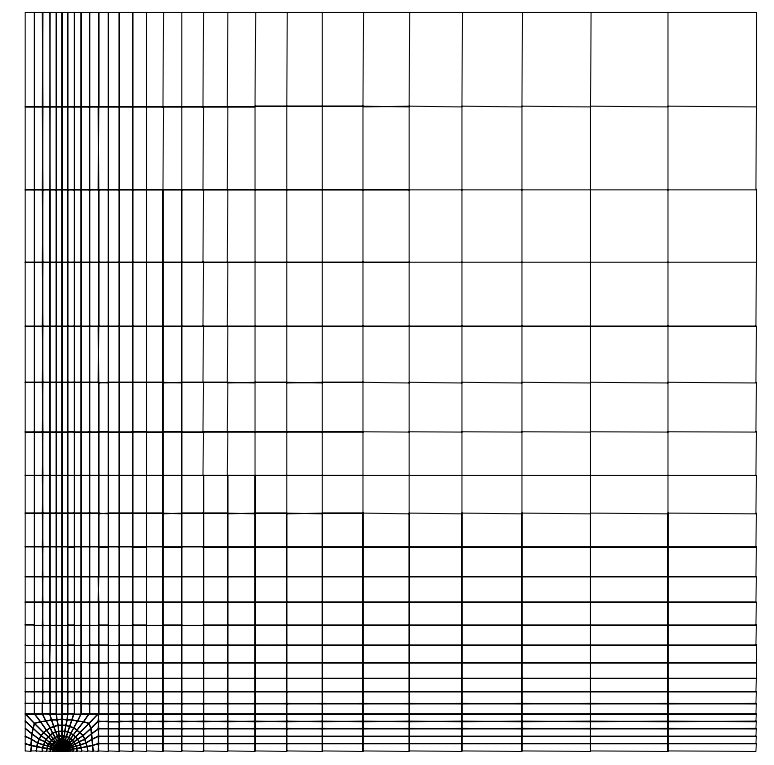

Fig.4. Finite element mesh for the first quadrant of the panel in Fig.5 


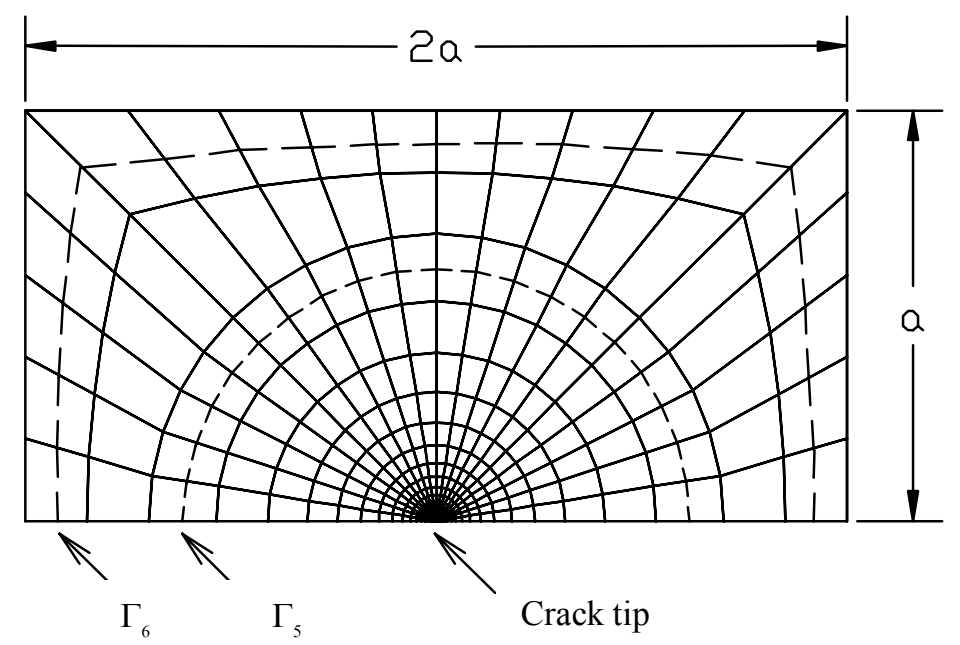

(a)

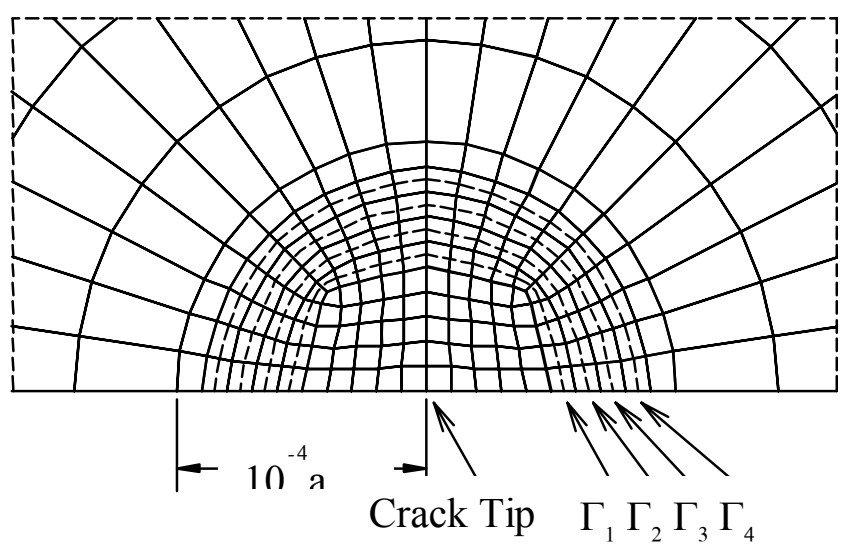

(b)

Fig.5. (a) Contours for global $J$-integral and (b) contours for local $J$-integral 


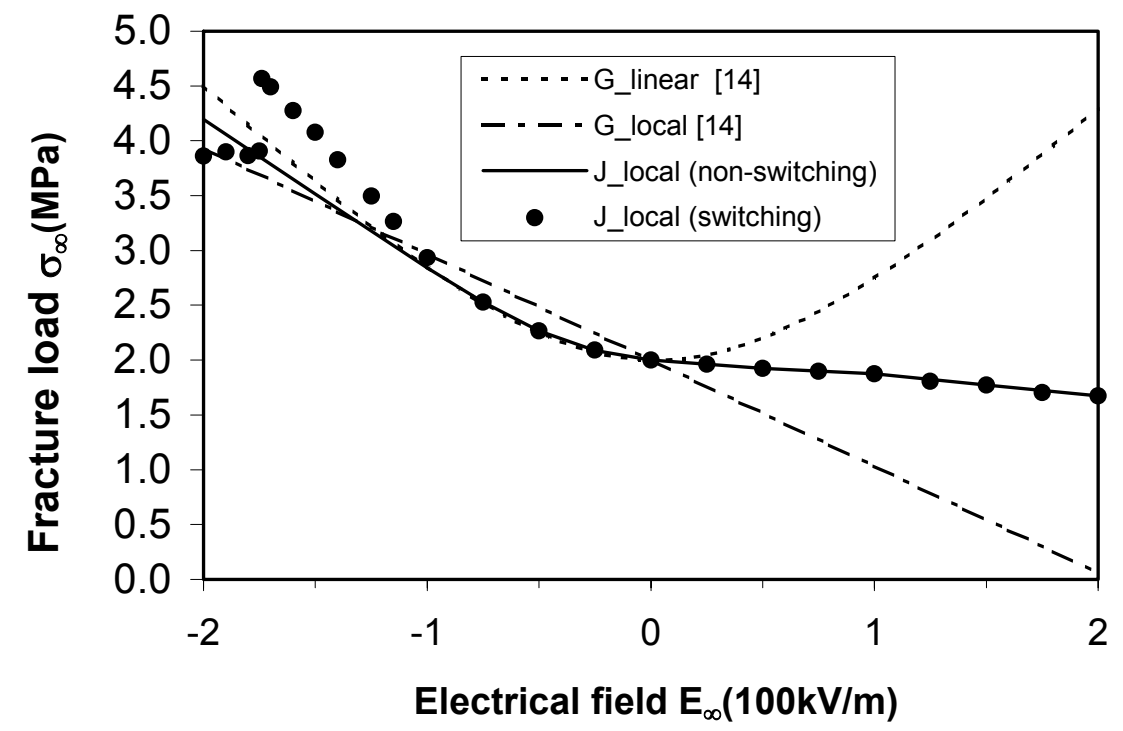

Fig.6. Relations between the fracture stress and the applied electric field by using $G_{\text {linear }}, G_{\text {local }}$ and $J_{\text {local }}$ as the fracture criteria 


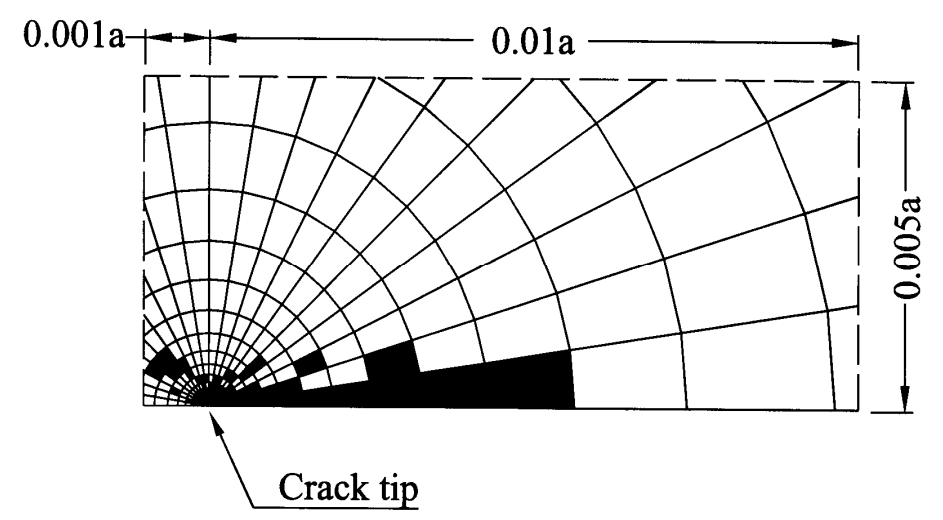

(a) $E_{\infty}=-175 \mathrm{kV} / \mathrm{m}$ and $\sigma_{\infty}=3.906 \mathrm{MPa}$

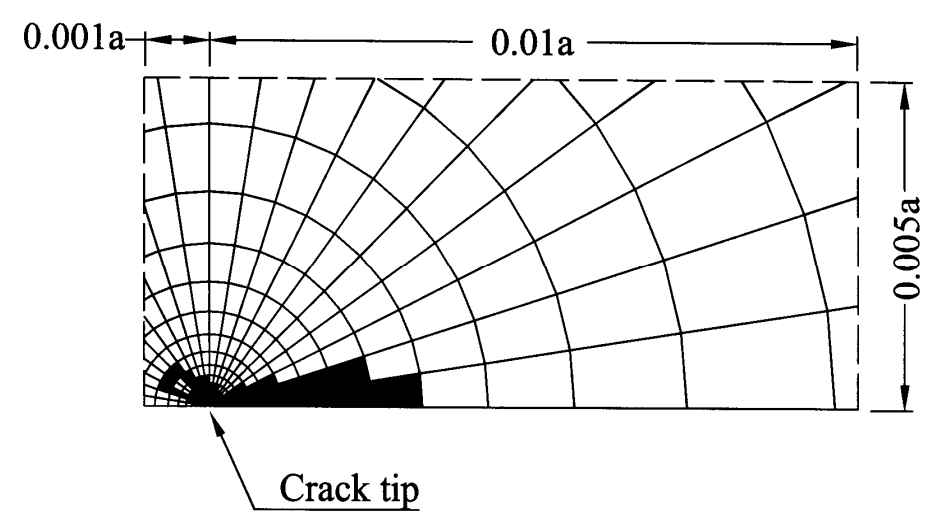

(b) $E_{\infty}=-174 \mathrm{kV} / \mathrm{m}$ and $\sigma_{\infty}=4.569 \mathrm{MPa}$

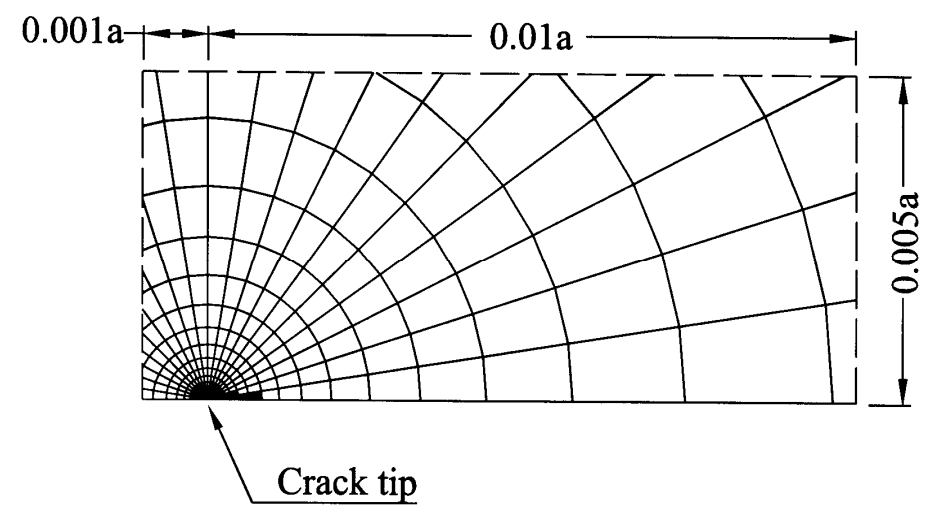

(c) $E_{\infty}=-100 \mathrm{kV} / \mathrm{m}$ and $\sigma_{\infty}=2.935 \mathrm{MPa}$ 

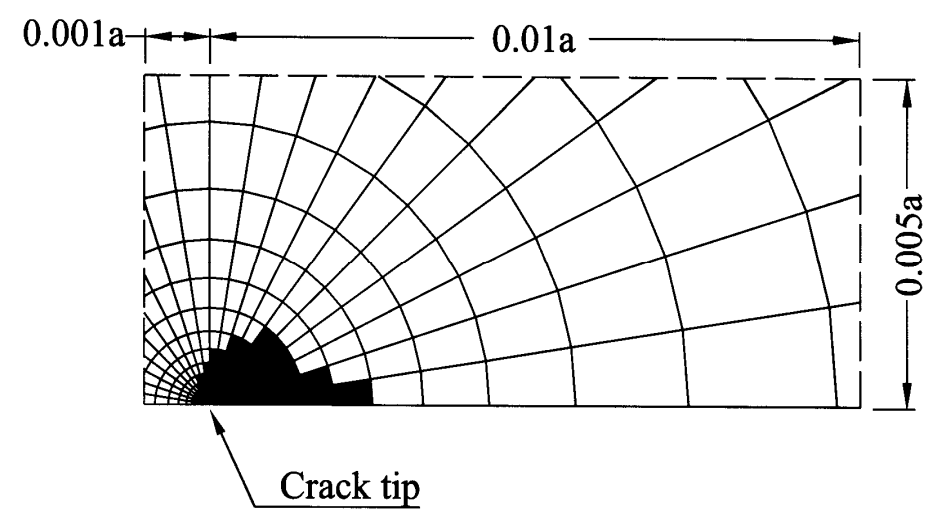

(d) $E_{\infty}=0 \mathrm{kV} / \mathrm{m}$ and $\sigma_{\infty}=2.000 \mathrm{MPa}$

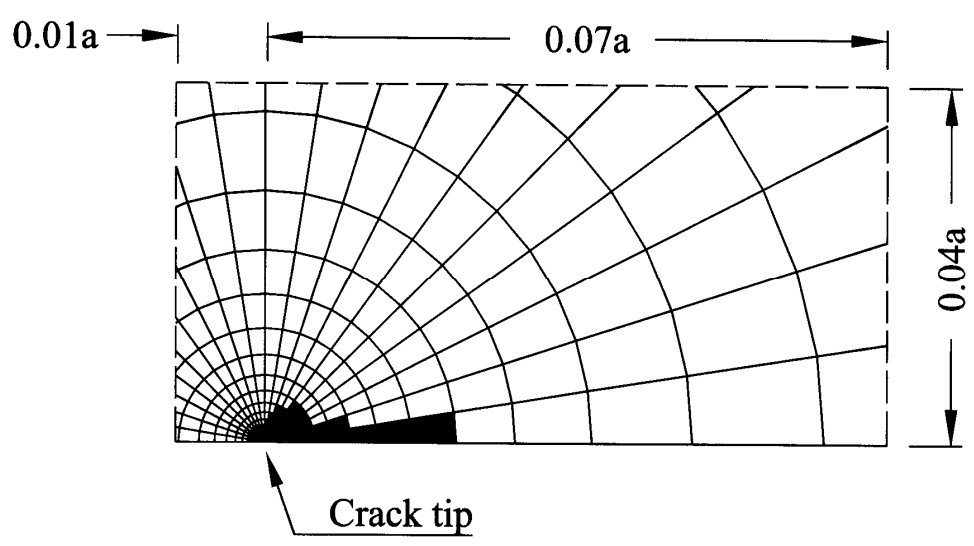

(e) $E_{\infty}=100 \mathrm{kV} / \mathrm{m}$ and $\sigma_{\infty}=1.874 \mathrm{MPa}$

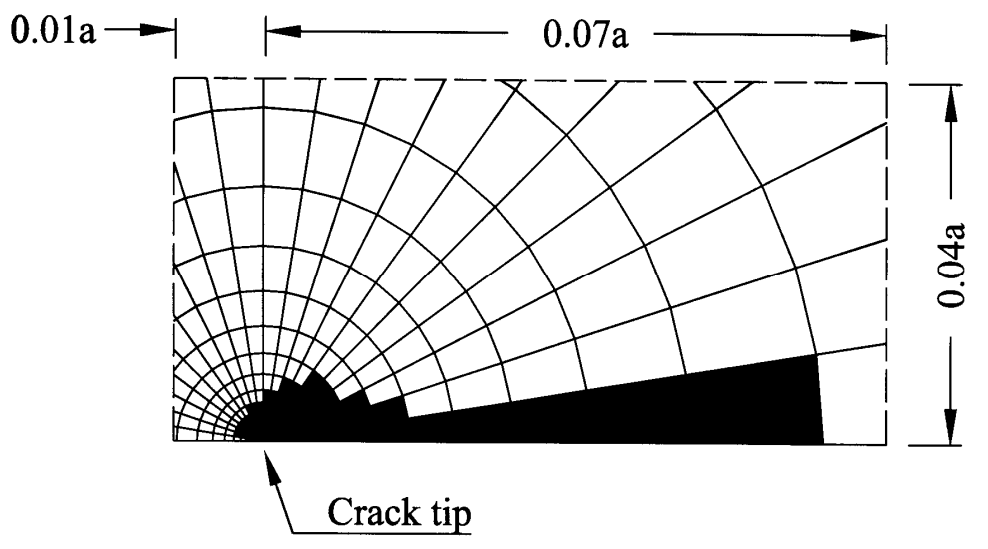

(f) $E_{\infty}=200 \mathrm{kV} / \mathrm{m}$ and $\sigma_{\infty}=1.675 \mathrm{MPa}$

Fig.7. Electric saturation zones predicted by using the switching model at different thresholds of cracking, see Fig.3a. 